\title{
LA COLABORACIÓN INTERUNIVERSITARIA EN CHILE. EL CASO DE PROYECTOS MECESUP DESDE LA PERSPECTIVA DE LAS CIENCIAS DE LA COMPLEJIDAD ${ }^{1}$
}

\section{Carolina Urbina ${ }^{2}$,Juan Pablo Cárdenas ${ }^{2,3}$, Darío Cárdenas ${ }^{2}$}

\section{RESUMEN}

Esta investigación analizó la colaboración entre universidades chilenas en el marco del programa MECESUP. Los resultados de la investigación pusieron de manifiesto que esta fue un fenómeno poco común y cada vez menos frecuente en este programa. Dentro de la pobre colaboración detectada, fueron solo algunas instituciones regionales del centro-sur de Chile las que tomaron un rol protagónico durante los últimos años, con redes de colaboración compuestas por unidades responsables de su ejecución cada vez más similares. Sin embargo, lo más sobresaliente de este estudio es que los resultados obtenidos parecen tener una estrecha relación con una medida de complejidad propuesta para las instituciones participantes en estos proyectos y la heterogeneidad del sistema de universidades estudiado.

Palabras clave: redes, colaboración universitaria, sistemas complejos, MECESUP

\section{COLLABORATION AMONG CHILEAN UNIVERSITIES. THE CASE OF MECESUP PROJECTS UNDER THE PERSPECTIVE OF COMPLEXITY SCIENCES}

\section{ABSTRACT}

This study has analysed the collaboration among Chilean universities in the program MECESUP. The results show that collaboration is an unusual phenomenon where regional institutions have taken a leading role in recent years in a process characterized by homogeneous networks of units responsible for projects implementation. However, most remarkable in this study is that results obtained seem to be related with a metric of complexity proposed for the institutions involved in this program and the heterogeneity of the studied system.

Keywords: networks, university collaboration, complex systems, MECESUP

1 Esta investigación ha contado con financiamiento del Consejo Nacional de Educación, Proyecto: Estudio de la Dinámica de la Red de Colaboración en Proyectos Adjudicados MECESUP2 del Fondo de Innovación Académica (2011).

2 Unidad de Innovación Educativa, Vicerrectoría Académica, Universidad de Santiago de Chile. Contacto: carolina.urbina@usach.cl

3 Instituto de Sistemas Complejos de Valparaíso. Subida Artillería 470, Valparaíso, Chile 


\section{LA COLABORACIÓN INTERUNIVERSITARIA EN CHILE. EL CASO DE PROYECTOS MECESUP DESDE LA PERSPECTIVA DE LAS CIENCIAS DE LA COMPLEJIDAD}

\section{Introducción}

Esta investigación se enmarca dentro del ámbito de las Ciencias de la Complejidad y su aplicación al estudio de la colaboración interuniversitaria en Chile.

El interés por atender a los procesos de colaboración viene dado por la creciente evidencia acerca del papel que tiene esta como medio fundamental para el fortalecimiento institucional, para el éxito de los organismos públicos y para la mejora de la calidad de la educación universitaria (Suurla, Markkula y Mustarjarvi, 2002; Sebastián, 2004; Jarvis, 2006; Meyer, Ramírez y Wotipka, 2008).

En consecuencia, el fundamento de la colaboración interuniversitaria, nacional e internacional, está basado en la complementariedad de las capacidades para realizar actividades en conjunto y así lograr mayor productividad, beneficios mutuos, visibilidad, progreso y mejoras de las capacidades competitivas de las instituciones, entre muchos otros aspectos.

Según Sebastián (2004), si se analiza la evolución en los modos de producción y gestión del conocimiento en los últimos años se observará la transición desde las investigaciones basadas en la individualidad de los científicos hacia aquellas basadas en los grupos de investigación, así como un mayor desarrollo de la colaboración entre grupos de diferentes instituciones y países y, últimamente, hacia algunas que se fundamentan en la constitución de redes de investigación, heterogéneas en su composición y transitorias en el tiempo.

La sociedad actual es una sociedad de redes en donde las funciones centrales están basadas cada vez más en dichos vínculos, por 
lo cual la participación y la dinámica del trabajo en red constituyen importantes fuentes de energía para su funcionamiento (Suurla, Markkula y Mustarjarvi, 2002). Es dentro de este panorama que parece interesante estudiar las relaciones de colaboración interuniversitaria que se han desarrollado en Chile en los últimos años.

Específicamente, en este estudio se analizó la colaboración interuniversitaria en uno de los concursos públicos impulsado por la División de Educación Superior del Ministerio de Educación: el Programa de Mejoramiento de la Calidad en la Educación Superior (MECESUP), particularmente en relación con los resultados de las tres convocatorias desarrolladas entre los años 2006 y 2008, correspondientes a la segunda etapa de dicho programa (MECESUP2).

Para estudiar dicha colaboración se utilizaron herramientas computacionales de la Ciencia de Redes Complejas (o simplemente Teoría de Redes) específicas para el análisis de la estructura relacional de los sistemas. Esta teoría es parte de las llamadas "Ciencias de la Complejidad" que se preocupan del estudio de sistemas complejos y de las leyes universales que gobiernan su funcionamiento.

La investigación comenzó con un análisis típico de la colaboración interuniversitaria desde la perspectiva de la Teoría de las Redes, es decir, caracterizando esa colaboración de acuerdo con los tipos de universidades participantes, sus vínculos y su dinámica durante el programa MECESUP2. No obstante, luego de una serie de reuniones y análisis de los resultados surgió el interés por explorar las posibles causas que ayudaran a comprender el comportamiento colaborativo observado. Para ello, se complementaron los análisis con los datos del programa MECESUP1 y se abordó el problema desde una perspectiva aún más profunda, en la búsqueda de posibles mecanismos subyacentes que explicaran lo observado; una mirada acorde con el estudio de los sistemas complejos que sugería que la colaboración detectada podía corresponder a un fenómeno emergente, no planificado y resultante de ciertas reglas muy básicas que operaban entre universidades.

Dicho esto, la investigación que se presenta se ha estructurado de la siguiente manera: en la primera sección se describen los 
principales elementos del programa MECESUP, así como otros estudios acerca de la colaboración que consideraron el problema desde una perspectiva similar. La metodología utilizada para analizar la colaboración se detalla en la segunda sección. En la tercera sección se ofrecen los principales resultados obtenidos del análisis inicial de la estructura colaborativa, presentando con mayor detalle aquellos referidos al programa MECESUP2. En la cuarta sección se muestra una hipótesis respecto del estado de la colaboración interuniversitaria en proyectos MECESUP. Finalmente, se describen las conclusiones y los principales comentarios de esta investigación.

\section{El programa MECESUP}

El programa MECESUP ha tenido como objetivo proveer a las instituciones de educación superior de las competencias necesarias que permitan incrementar su competitividad internacional, sostener el desarrollo económico y social del país, y asegurar la calidad y la equidad en el sistema de educación superior (MECESUP2, 2011).

De manera específica, MECESUP ha entregado financiamiento a proyectos y planes de mejoramiento institucional adjudicados por medios competitivos, o bien, negociados a través del Fondo de Innovación Académica (FIAC) y de los Convenios de desempeño. Hasta la fecha dicho programa ha asignado cerca de 770 proyectos con el apoyo del Banco Mundial.

En la llamada "fase 1" de los proyectos MECESUP (1999-2005), el programa operó bajo la hipótesis general de que la inversión en infraestructura académica mejoraría la docencia y el aprendizaje estudiantil. Debido a esto, gran parte de los proyectos adjudicados se desarrollaron en torno al reforzamiento de las capacidades base de las instituciones de educación superior, tales como la infraestructura y el equipamiento, la renovación de programas y la renovación de personal académico.

En el año 2005 comenzó la segunda fase del programa, llamada MECESUP2. Esta segunda fase, que es la que se estudió en mayor profundidad en esta investigación, involucró un nuevo convenio 
con el Banco Mundial y complementó el Fondo Competitivo con la implementación experimental de convenios de desempeño en un número limitado de universidades acreditadas del Estado. El Fondo Competitivo (llamado desde ese momento Fondo de Innovación Académica) tuvo tres llamados a concurso (2006, 2007 y 2008) y adjudicó 351 proyectos. Esta segunda etapa del MECESUP promovió los procesos de innovación respecto de la armonización curricular, los programas de doctorado y las redes colaborativas.

La siguiente investigación centró su atención en los proyectos adjudicados mediante el Fondo de Innovación Académica (FIAC), cuyos ejes temáticos aparecen descritos en la siguiente tabla:

Tabla 1: Ejes temáticos para el programa MECESUP2.

\begin{tabular}{|l|l|}
\hline Convocatoria & $\begin{array}{l}\text { Formación de capital humano avanzado. } \\
\text { Innovación académica. } \\
\text { Mejoramiento de la gestión académica. } \\
\text { Renovación curricular. }\end{array}$ \\
\hline Convocatoria & $\begin{array}{l}\text { Desarrollo de personal académico y gestión docente. } \\
\text { Desarrollo de programas de doctorados nacionales. } \\
2007\end{array}$ \\
$\begin{array}{l}\text { Mejoramiento de los resultados docentes. } \\
\text { Renovación curricular basada en resultados de aprendizaje y competencias. } \\
\text { Mejoramiento de la oferta de formación técnica de nivel superior. }\end{array}$ \\
\hline $\begin{array}{l}\text { Convocatoria } \\
2008\end{array}$ & $\begin{array}{l}\text { Desarrollo de personal académico para la investigación y para la gestión. } \\
\text { Desarrollo de programas de postgrado nacionales. } \\
\text { Mejoramiento de los resultados docentes. } \\
\text { Modernización curricular basada en resultados de aprendizaje y competencias. }\end{array}$ \\
\hline
\end{tabular}

En la actualidad, MECESUP está aplicando un nuevo formato de convenios de desempeño para el fortalecimiento institucional de los Centros de Formación Técnica y los Institutos Profesionales, apoyo para el fortalecimiento y renovación de las carreras de pedagogía, competitividad de la formación técnica y el sector productivo, entre otros (MECESUP2, 2011).

En el marco del programa MECESUP2, las instituciones de educación superior se visualizan como uno de los principales responsables en la formación de personas para la nueva sociedad del conocimiento. Por ello, es fundamental reformar el sistema de educación terciaria para posicionarlo a la altura de la tarea que la sociedad le ha entregado. Desde los mismos fundamentos del programa 
MECESUP2 ha sido clara la relevancia de la integración de una red global de instituciones de educación terciaria, las garantías de la calidad de la formación, la investigación que realiza y el rediseño de los mecanismos de certificación de los títulos profesionales que entregan.

Según Sebastián (2004) dos de los indicadores que evidencian más nítidamente la tendencia del predominio de formas organizativas sustentadas en la colaboración son, por una parte, aquellos relacionados con la composición de los proyectos y redes y, por otra, los indicadores bibliométricos.

Es precisamente desde esta perspectiva que Olmeda y colaboradores (2009) estudiaron los patrones de colaboración interuniversitaria en España por medio del análisis de indicadores bibliométricos, usando para esto la Teoría de Redes, un enfoque similar al adoptado en esta investigación. En particular, dichos autores analizaron la estructura de las redes de colaboración de universidades estatales y privadas, considerando sus cooperaciones en publicaciones científicas.

La investigación tuvo interesantes resultados: por un lado, identificó la dependencia de universidades periféricas de otras centrales en España, destacando la influencia de la proximidad geográfica en la elección de socios para la colaboración por sobre la calidad científica de estos; y, por otro, se constató la creciente internacionalización de la investigación universitaria española. Por ejemplo, dicha investigación puso en evidencia que más del 85\% de la producción universitaria de artículos con colaboración internacional se firma con socios de la Unión Europea, mientras que un 30\% lleva coautoría norteamericana.

En Chile, durante el año 2009, se desarrolló una investigación que indagó respecto de la estructura en las redes de colaboración e intercambio de información en la Facultad de Ciencias Sociales de la Pontificia Universidad Católica de Chile (García, 2009). Haciendo uso también de la Teoría de Redes, esta investigación quiso visualizar las redes en función del flujo de información (específicamente vía correos electrónicos) y de colaboración científica (según el número 
de artículos publicados). Como resultado, el estudio demostró cómo la estructura formal de la universidad determinaba las formas de coordinación y la posibilidad de emergencia de colectivos colegiados y colaborativos. Por ejemplo, identificó diferentes roles entre profesores y autoridades administrativas en el sistema de correo electrónico, de manera que en la red general los profesores ocupaban una posición aparentemente pasiva, mientras que las autoridades administrativas se posicionaban como distribuidores y centros de actividad de la red. Por otro lado, corroboró que las actividades de publicación estaban entregando reconocimiento y estatus a sus autores, de acuerdo con lo analizado respecto de los flujos de correo electrónico y el análisis de publicaciones científicas.

Según Sebastián (2004) la complementariedad y la colaboración son cada vez más necesarias en un mundo donde más disciplinas científicas, así como las propias tecnologías, se fusionan. A esto se debe sumar la creciente especialización de los grupos de investigación y el carácter multidisciplinar de los estudios, razón por la cual es evidente la importancia que cobra la colaboración en la gestión actual del conocimiento.

\section{Estudio de la colaboración interuniversitaria en el programa MECESUP desde la perspectiva de la Teoría de Redes}

El reduccionismo ha dominado la ciencia y la filosofía por siglos, sin embargo, ese panorama está cambiando rápidamente. En la actualidad, gracias a la evidencia aportada por las Ciencias de la Complejidad y, en particular, por la Teoría de Redes, se sabe que las interacciones de un sistema -aquellas que el reduccionismo no considera- son fundamentales para entender ciertos fenómenos como, por ejemplo, la colaboración. Es más, cuando las interacciones de un sistema son consideradas, el reduccionismo aparece limitado en varios aspectos (Gershenson, 2012).

En consecuencia, utilizando un conjunto de algoritmos computacionales para visualizar y caracterizar redes, similares a los usados por las investigaciones antes mencionadas (Olmeda et al., 2009 
y García, 2009), en esta investigación se estudiaron las propiedades de las relaciones de colaboración (interacciones) generadas en cada año del programa MECESUP, para los distintos ejes temáticos. Además, se estudió la dinámica de dichas relaciones de colaboración en el marco de dicho programa.

Para desarrollar esta tarea se contó con la colaboración de la División de Educación Superior del MINEDUC, entidad que facilitó los datos necesarios para el estudio. Además, durante el proceso de investigación se sostuvieron entrevistas con informantes clave tanto del MINEDUC, como de otras instituciones de educación superior, quienes colaboraron en el análisis de los resultados y en las reflexiones respecto de las contribuciones que un estudio de este tipo podría aportar.

En términos técnicos, para construir las redes de colaboración se trasformaron los proyectos MECESUP, ejecutados en colaboración, en grafos del tipo $G(\mathrm{~N}, \mathrm{E})$. Estos grafos describieron la relación entre $N$ universidades a través de $E$ enlaces. Para establecer las relaciones de colaboración entre las universidades se consideró que: dos universidades colaboran si es que se han adjudicado en forma conjunta uno o más proyectos MECESUP en alguno de los años del programa.

Por ejemplo, la Figura 1 muestra la representación más básica de un grafo de colaboración (red básica) considerando un grupo de ocho universidades. Así, los E=21 enlaces entre las universidades representarían relaciones de colaboración y cada universidad representaría un nodo de la red. En dicha representación se observa que la mayoría de estas universidades mantiene un número similar de colaboraciones, a excepción de la Universidad Austral de Chile (UACH) que colabora solamente con la Universidad de Concepción (UDEC). 
Figura 1: Ejemplo de red básica de colaboración entre 8 universidades $G(8,21)$

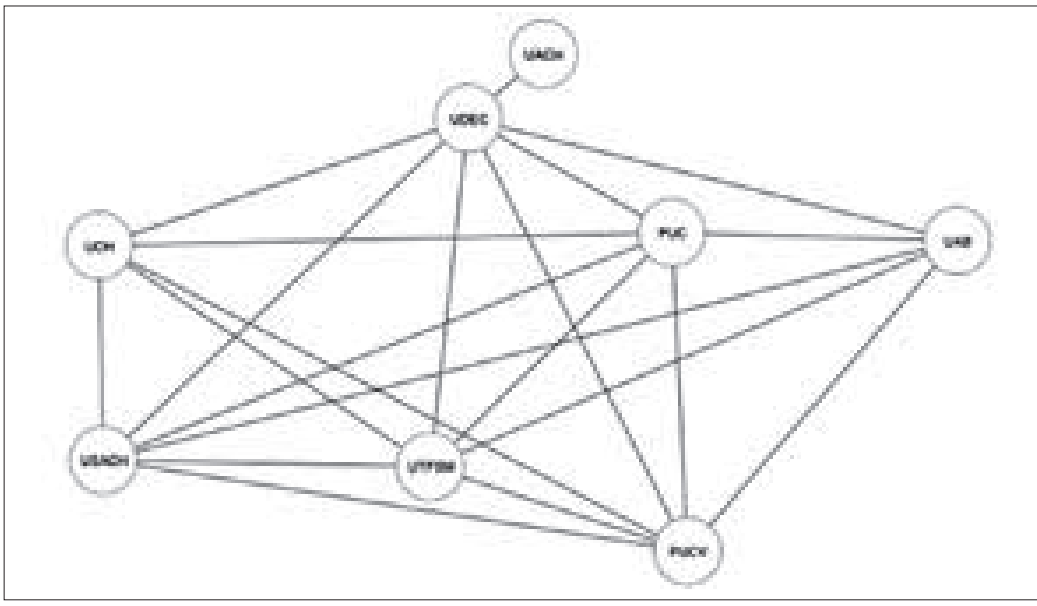

Otra representación de las relaciones de colaboración se verificó por medio de grafos pesados, los cuales contienen información específica de los enlaces. Por ejemplo, la Figura 2 muestra un grafo en donde se observan las relaciones entre universidades según el monto de dinero asignado al proyecto adjudicado en común (lo que sería una propiedad del enlace). De este modo, se visualizan relaciones menos fuertes entre la Universidad de Chile (UCH) y la Universidad de Santiago (USACH), en comparación con las que tiene la Universidad Andrés Bello (UAB) con la Universidad de Concepción (UDEC) o la Universidad Católica de Valparaíso (PUCV).

Figura 2: Ejemplo de grafo pesado. El grosor de los enlaces indica el monto de dinero asignado al proyecto adjudicado en colaboración

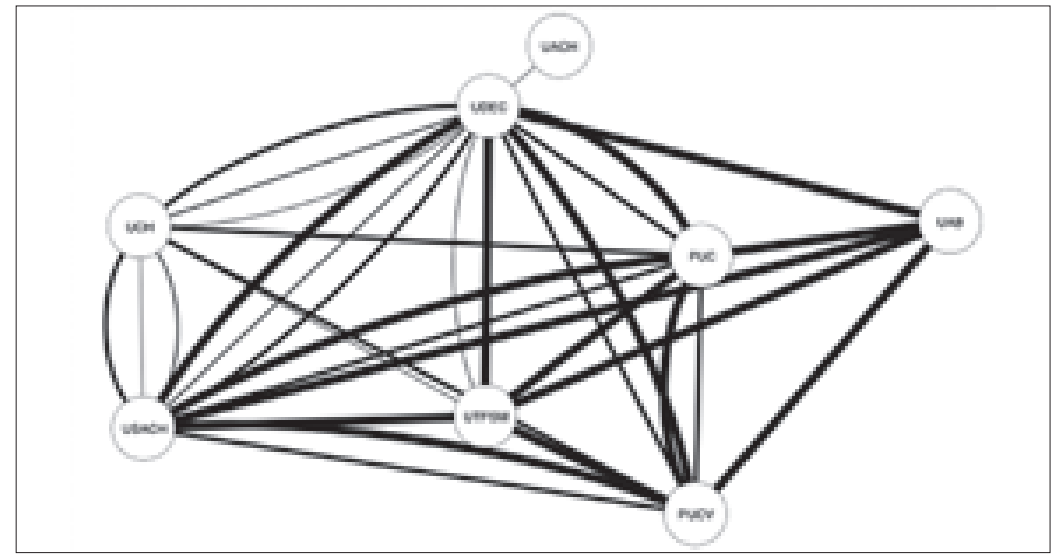


Además de lo anterior, para la construcción de las redes de colaboración se consideraron ciertos atributos de las universidades (nodos). Entre estos se contempló el tipo de administración, la filiación religiosa y la ubicación geográfica.

Respecto del tipo de administración, las universidades fueron clasificadas como estatales, particulares o privadas, de acuerdo con la clasificación utilizada por el MINEDUC. Se consideró estatales a aquellas que pertenecen al Consejo de Rectores y que se financian principalmente con el aporte directo del Estado; como particulares a aquellas que pertenecen al Consejo de Rectores, pero no son de propiedad estatal; $y$, por último, como universidades privadas a aquellas que funcionan solo con aportes privados.

Considerando su filiación religiosa, las universidades se clasificaron en laicas, pertenecientes a la Iglesia Católica, diocesanas ${ }^{4}$ y pertenecientes a una orden religiosa ${ }^{5}$. Respecto de la ubicación geográfica, las universidades se clasificaron en regionales o metropolitanas.

La Figura 3 muestra la misma red de la Figura 1, pero con sus nodos caracterizados según el tipo de administración y la filiación religiosa. Cada atributo se representó con un tono y una forma particular.

4 Aquellas instituciones que siguen los valores de la Iglesia y que pertenecen a una determinada Diócesis, zona geográfica en la que la Iglesia peregrina.

5 Organización aprobada por la Iglesia a través de ciertos votos, tal como los Jesuitas o los Salesianos. 
Figura 3: Ejemplo de red de colaboración caracterizada según el tipo de administración y filiación religiosa. El tipo de administración se representa según la forma de cada nodo de la red (círculo=Estatales, diamante=Particulares, V=Privadas). La filiación religiosa se representa según el tono del nodo en la red (claro=Laicas, oscuro=de la Iglesia)

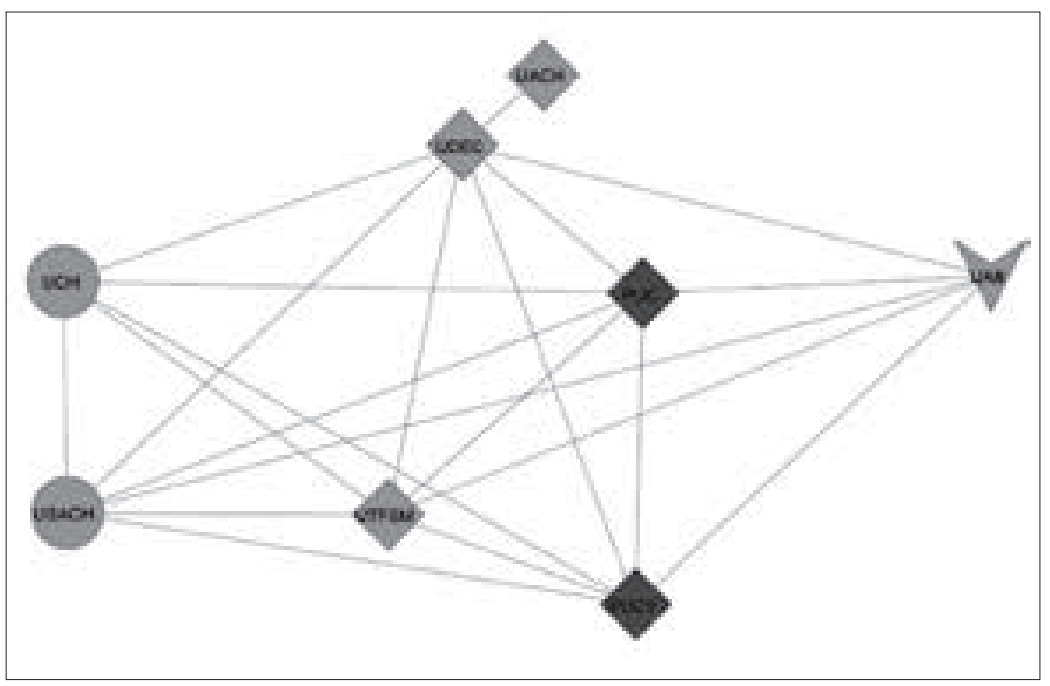

En el ejemplo de la Figura 3 se observa que la mayoría de las universidades de la red son particulares, dos de ellas son estatales y solo una es privada. También se aprecia que la mayoría de las universidades son laicas, excepto la Pontificia Universidad Católica de Chile (PUC) y la Pontificia Universidad Católica de Valparaíso (PUCV), que pertenecen a la Iglesia.

Con otro tipo de análisis se obtuvo información acerca de ciertas propiedades estructurales de las redes de colaboración, así como también de las propiedades de las universidades en el contexto de la red a la que pertenecen. Respecto de estas últimas, considerando las relaciones de colaboración, se caracterizó a las universidades según su grado de colaboración y según su posición estratégica en las redes. El primer factor corresponde a la cantidad de enlaces de colaboración que una universidad posee en una determinada red. Por su parte, la posición estratégica de colaboración refleja aquella conectividad que le permite a una universidad ser el puente de comunicación entre universidades que no estarían conectadas en su ausencia. 
A nivel estructural de la red, se calcularon propiedades tales como la distribución de conectividades y la densidad de enlaces. La distribución de conectividades indica qué tan heterogénea es la colaboración en una determinada red, mientras que la densidad refleja cuán poblada de colaboración está esa red.

Finalmente, el estudio de la colaboración se profundizó por medio de un análisis complementario de mayor resolución que consideró a las unidades responsables en la ejecución de proyectos MECESUP como nodos de la red. En este caso, los nodos pasaron a ser las Vicerrectorías, Facultades y Escuelas, entre otras. Para realizar este análisis, las unidades responsables se clasificaron en siete grupos: de ciencias, de ingeniería, de educación, de humanidades, de arquitectura y diseño, de salud y unidades institucionales (tales como Vicerrectorías).

El objetivo de este análisis fue identificar los tipos de unidades responsables más frecuentes, así como también la diversidad de dichas unidades en las redes de colaboración. Así, se calculó la diversidad como una medida de heterogeneidad de unidades responsables presentes en una red. En consecuencia, una red diversa sería aquella formada por distintos tipos de unidades responsables, o bien, una homogénea, en caso de lo contrario.

\section{Resultados}

Los resultados que se presentan a continuación corresponden a los principales obtenidos del análisis de cada una de las redes de colaboración según la metodología descrita anteriormente. Para conocer mayor información acerca de estos y otros resultados, se sugiere revisar el Informe Final ${ }^{6}$ de esta investigación, publicado en la página web del Consejo Nacional de Educación.

6 "Estudio de la Dinámica de la Red de Colaboración en Proyectos Adjudicados MECESUP2 del Fondo de Innovación Académica (2011)". 


\subsection{Panorama general del programa MECESUP2 y su relación con programa MECESUP1}

Durante el programa MECESUP2 se financiaron en total 351 proyectos. De ellos, 47 fueron asignados en colaboración, lo que correspondió a un $13 \%$ del total de universidades participando en el proceso (28 instituciones). En la Tabla 2 se presentan las universidades participantes durante este periodo, así como su vinculación con proyectos de colaboración en cada uno de los años del periodo.

Tabla 2: Universidades que se han adjudicado proyectos en colaboración durante el programa MECESUP2 (periodo 2006-2008). En cada año aparece el número total de proyectos adjudicados por la universidad $y$, entre paréntesis, se indica el número de proyectos adjudicados en colaboración

\begin{tabular}{|l|c|c|c|c|}
\hline Universidad & Identificador & 2006 & 2007 & 2008 \\
\hline Universidad Técnica Federico Santa María & UTFSM & $6(4)$ & $13(2)$ & $12(2)$ \\
\hline Universidad de Chile & UCH & $14(8)$ & $14(4)$ & $6(0)$ \\
\hline Universidad de Concepción & UDEC & $13(7)$ & $10(5)$ & $3(1)$ \\
\hline Universidad de Santiago de Chile & USACH & $11(8)$ & $11(2)$ & $6(0)$ \\
\hline Pontificia Universidad Católica de Valparaíso & PUCV & $12(6)$ & $14(4)$ & $10(2)$ \\
\hline Pontificia Universidad Católica de Chile & PUC & $13(5)$ & $18(5)$ & $9(0)$ \\
\hline Universidad Andrés Bello & UAB & $3(1)$ & $0(0)$ & $2(0)$ \\
\hline Universidad Austral de Chile & UACH & $6(4)$ & $10(3)$ & $7(0)$ \\
\hline Universidad del Biobío & UBB & $11(10)$ & $9(3)$ & $2(1)$ \\
\hline Universidad de Valparaíso & UVA & $7(5)$ & $7(2)$ & $9(3)$ \\
\hline Universidad de La Frontera & UFRO & $8(5)$ & $6(3)$ & $2(1)$ \\
\hline Universidad de Talca & UTAL & $7(6)$ & $4(2)$ & $7(1)$ \\
\hline Universidad de La Serena & ULS & $5(5)$ & $2(1)$ & $1(0)$ \\
\hline Universidad de Tarapacá & UTA & $8(3)$ & $5(2)$ & $6(0)$ \\
\hline Universidad Metropolitana de Ciencias de la Educación & UMCE & $5(5)$ & $1(0)$ & $2(1)$ \\
\hline Universidad de Antofagasta & UANTOF & $4(3)$ & $5(1)$ & $6(2)$ \\
\hline Universidad de Playa Ancha & UPLA & $5(4)$ & $2(0)$ & $2(1)$ \\
\hline Universidad de Los Lagos & ULAGOS & $4(3)$ & $2(0)$ & $6(3)$ \\
\hline Universidad Arturo Prat & UNAP & $3(2)$ & $2(0)$ & $1(1)$ \\
\hline Universidad de Magallanes & UMAG & $5(4)$ & $6(1)$ & $3(1)$ \\
\hline Universidad de Atacama & UDA & $2(2)$ & $3(2)$ & $0(0)$ \\
\hline Universidad Católica del Norte & UCN & $7(2)$ & $9(2)$ & $5(0)$ \\
\hline Universidad Católica del Maule & UCM & $5(4)$ & $2(1)$ & $3(0)$ \\
\hline Universidad Católica de Temuco & UCT & $6(4)$ & $8(3)$ & $7(4)$ \\
\hline Universidad Católica Cardenal Silva Henríquez & UCSH & $1(1)$ & $2(0)$ & $0(0)$ \\
\hline Universidad Alberto Hurtado & UAH & $1(1)$ & $3(0)$ & $1(0)$ \\
\hline Universidad Católica de la Santísima Concepción & UCSC & $2(2)$ & $8(3)$ & $3(2)$ \\
\hline Universidad Tecnológica Metropolitana & UTEM & $3(2)$ & $2(1)$ & $3(0)$ \\
\hline
\end{tabular}


Por su parte, en la Tabla 3 se presenta la descripción de las universidades participantes en proyectos de colaboración en función de los atributos considerados en este estudio, vale decir: tipo de administración, filiación religiosa y ubicación geográfica. Tal como se puede apreciar, la mayor parte de las universidades que se adjudicaron proyectos MECESUP2 en colaboración fueron estatales, laicas y regionales.

Tabla 3: Universidades participantes según tipo de administración, filiación religiosa y ubicación geográfica

\begin{tabular}{|l|c|c|c|}
\hline Universidad & Tipo de administración & Filiación religiosa & Ubicación geográfica \\
\hline UTFSM & Particular & Laica & Regional \\
\hline UCH & Estatal & Laica & Metropolitana \\
\hline UDEC & Particular & Laica & Regional \\
\hline USACH & Estatal & Laica & Metropolitana \\
\hline PUCV & Particular & Iglesia & Regional \\
\hline PUC & Particular & Iglesia & Metropolitana \\
\hline UAB & Privada & Laica & Metropolitana \\
\hline UACH & Particular & Laica & Regional \\
\hline UBB & Estatal & Laica & Regional \\
\hline UVA & Estatal & Laica & Regional \\
\hline UFRO & Estatal & Laica & Regional \\
\hline UTAL & Estatal & Laica & Regional \\
\hline ULS & Estatal & Laica & Regional \\
\hline UTA & Estatal & Laica & Regional \\
\hline UMCE & Estatal & Laica & Metropolitana \\
\hline UANTOF & Estatal & Laica & Regional \\
\hline UPLA & Estatal & Laica & Regional \\
\hline ULAGOS & Estatal & Laica & Regional \\
\hline UNAP & Estatal & Laica & Regional \\
\hline UMAG & Estatal & Laica & Regional \\
\hline UDA & Estatal & Laica & Regional \\
\hline UCN & Particular & Diocesana & Regional \\
\hline UCM & Particular & Diocesana & Regional \\
\hline UCT & Particular & Diocesana & Regional \\
\hline UCSH & Privada & Orden Religiosa & Metropolitana \\
\hline UAH & Privada & Orden Religiosa & Metropolitana \\
\hline UCSC & Diocesana & Regional \\
\hline UTEM & Laica & Metropolitana \\
\hline
\end{tabular}

Ahora bien, para comenzar el análisis de la colaboración interuniversitaria, se construyeron redes básicas para cada año del programa MECESUP2 y en cada año se consideraron todos los proyectos y ejes temáticos. 
Figura 4: Redes básicas de colaboración del programa MECESUP2. Año 2006 izquierda arriba, año 2007 derecha arriba y año 2008 abajo

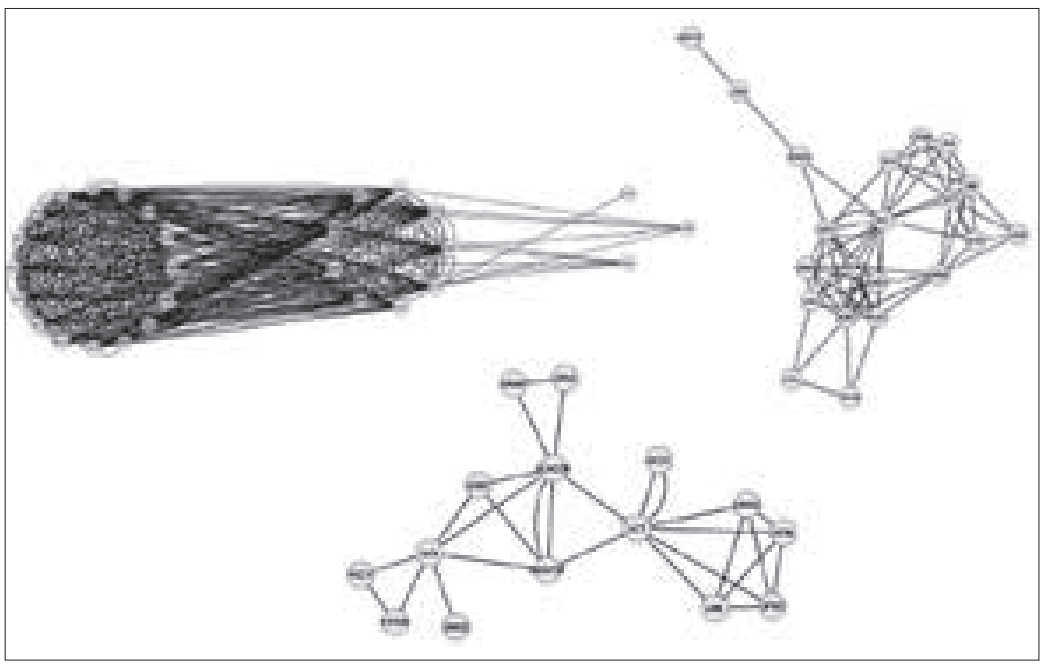

En la Figura 4 se puede apreciar la conformación básica de cada una de las redes de colaboración construidas para cada año de ejecución del programa MECESUP2. Se observa claramente que el número de enlaces disminuye a medida que transcurren los años: la red básica de colaboración del año 2006 fue más densa, es decir, más poblada de enlaces, que la red básica de colaboración del año 2007 y mucho más densa que la del año 2008. Lo anterior indica una disminución progresiva de la colaboración interuniversitaria en el programa MECESUP2.

Para profundizar este análisis, se comparó el número de universidades participantes, número de enlaces (colaboración) y densidad de colaboración en cada uno de los años del programa MECESUP2 (Figura 5). Se observó que la disminución de la colaboración (enlaces) también estuvo acompañada de un descenso del número de universidades participantes. Esto indica que las redes no solo presentaron menos colaboración, sino que también cada vez fue menor el número de universidades que se adjudicaron proyectos en red. 

MECESUP DESDE LA PERSPECTIVA DE LAS CIENCIAS DE LA COMPLEJIDAD - C. Urbina, J. P. Cárdenas, D. Cárdenas

Figura 5: Dinámica de la colaboración interuniversitaria durante el programa MECESUP2: densidad de colaboración (arriba), número de colaboraciones (medio) y universidades participantes en colaboración (abajo)

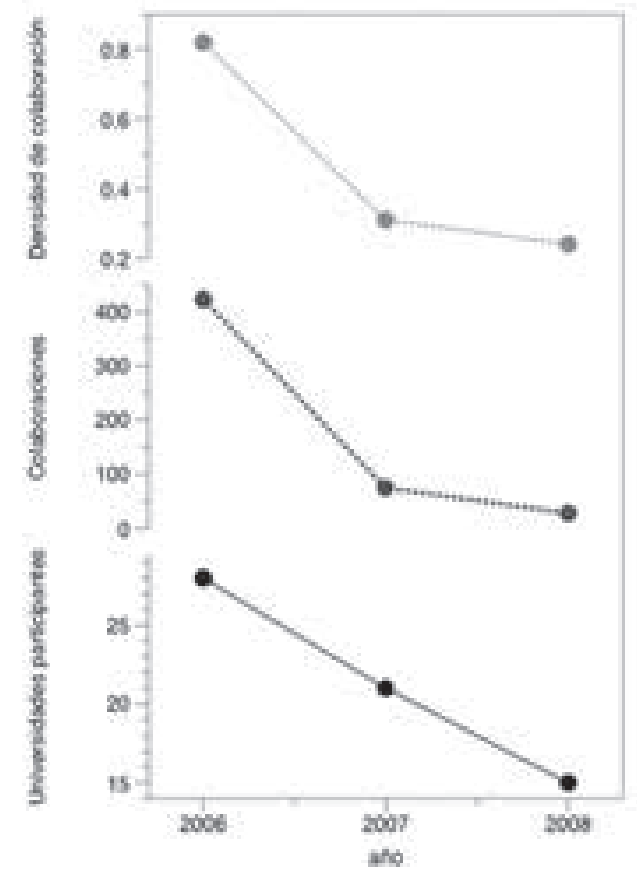

Con ello, se puede señalar que hubo una transición desde una red de colaboración interuniversitaria en la cual prácticamente todas las universidades colaboraban en conjunto (año 2006) a redes con menor cantidad y densidad de colaboración.

Al analizar la cantidad de proyectos en colaboración adjudicados durante el programa MECESUP2 también se observó una tendencia a la disminución. En el año 2006 un 25\% del total de proyectos adjudicados incluyó una relación colaborativa, en tanto que en el año 2007 este porcentaje bajó a un 11\%, llegando al año 2008 con un porcentaje que apenas ascendió a un 8\%.

Considerando este escenario, se exploró la posibilidad de que esta fuera una tendencia heredada desde el programa MECESUP1. Para ello se analizaron los datos de dicho programa en todos sus años (1999-2004) utilizando la misma metodología aplicada para los 
del MECESUP2. Los resultados generales de este análisis se pueden observar en la siguiente figura.

Figura 6: Panorama de la colaboración durante el programa MECESUP1

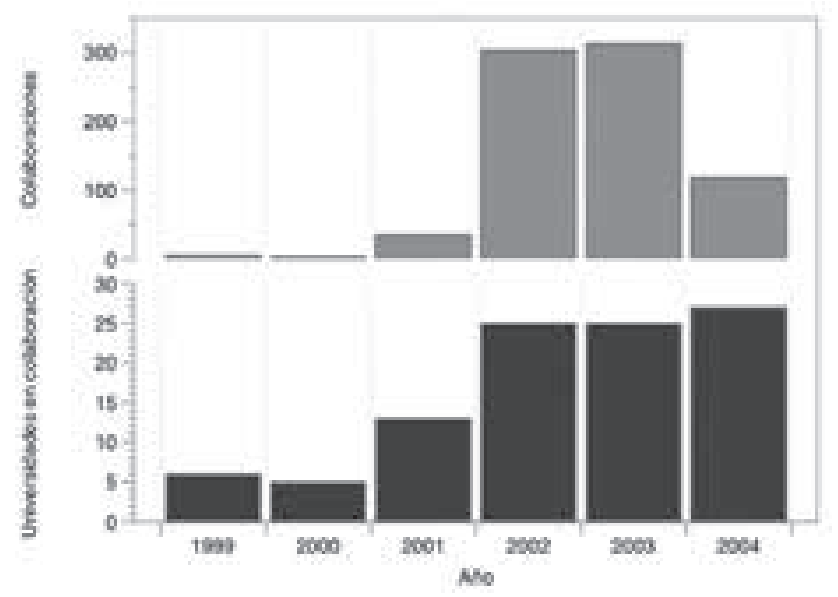

Se apreció que durante el programa MECESUP1 la colaboración en los tres primeros años fue baja, mientras que en los años 2002 y 2003 hubo un alza considerable, que luego cayó al año siguiente. Respecto de las universidades, se apreció un alza sostenida en el número de instituciones participantes en relaciones de colaboración, a excepción de lo observado en el año 2000. 
Figura 7: Panorama general de la colaboración en programas MECESUP

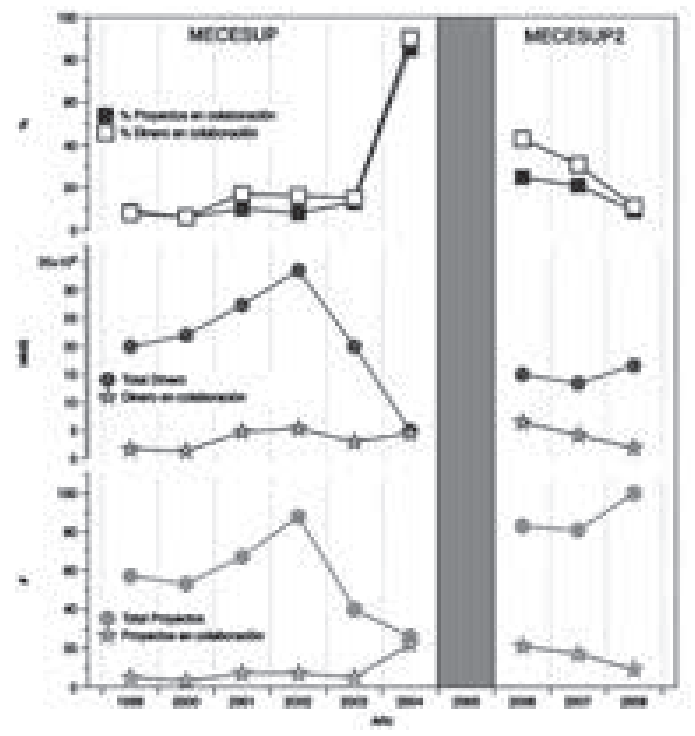

El panorama de la colaboración interuniversitaria en ambos periodos del programa MECESUP se muestra en la Figura 7. Se observa que durante la segunda fase hubo un alza significativa en el número de proyectos totales adjudicados (gráfica inferior, línea con círculos) aunque con menores montos asignados (gráfica media). Sin embargo, llama la atención la tendencia a la disminución de las relaciones de colaboración (línea roja con estrellas en el gráfico inferior) que pareciera ser un comportamiento heredado desde la primera fase, por lo menos en cantidad de proyectos ejecutados en colaboración.

\subsection{Colaboración en el programa MECESUP2}

El análisis anterior da cuenta de uno de los principales resultados de este estudio: la tendencia hacia una ejecución cada vez más individual de proyectos MECESUP. No obstante, y a pesar del panorama de pobre colaboración, aparecen otros resultados llamativos que pueden complementar el análisis.

Uno de estos dice relación con el tipo de universidades que jugaron roles protagónicos en la colaboración durante este programa. De acuerdo con el atributo de ubicación geográfica, durante el 
programa MECESUP2 fueron cuatro universidades regionales del centro-sur del país (UDEC, UFRO, ULAGOS y UCT) las que aparecen asumiendo esos roles, especialmente al final de esta fase, tanto por el número de colaboraciones que generaron, como por la posición estratégica que adoptaron dentro de las redes. Destaca dentro de este grupo la UDEC, también protagonista durante MECESUP1. Dentro de las universidades metropolitanas, la USACH fue la más colaborativa, tomando el rol que desempeñaron la UCH y la PUC durante la primera fase.

Según otros atributos de las universidades, los resultados mostraron que la colaboración en cada año se manifestó más frecuentemente entre instituciones estatales y laicas. Sin embargo, cuando se analizaron las redes a nivel de cada eje temático, el protagonismo en la colaboración lo tuvieron las universidades particulares y diocesanas. Esta diferencia se debe a que las redes construidas para cada eje temático corresponden a una parte de aquella red global que considera todos los ejes durante un año. Esto altera completamente la estructura de conectividad.

Respecto de la responsabilidad en el liderazgo en los proyectos colaborativos, los resultados mostraron que el $77 \%$ de los proyectos que generaron mayor colaboración (más universidades trabajando en conjunto) fueron dirigidos por universidades regionales, lo cual remarca el importante rol que jugaron estas instituciones en la colaboración durante el programa MECESUP2, algo totalmente distinto de lo observado durante la primera fase. Es más, si solo se consideran los años 2007 y 2008, todos los proyectos con mayor colaboración fueron dirigidos por universidades regionales.

Finalmente, el análisis de la colaboración entre unidades responsables de los proyectos mostró que aquellas de carácter "institucional" (como Vicerrectorías y unidades administrativas, por ejemplo) fueron las que participaron en más proyectos colaborativos. En términos de la diversidad de los tipos de unidades, los resultados mostraron que las redes fueron cada vez más homogéneas, es decir, compuestas por unidades responsables similares. 
En resumen, el análisis mostró que la colaboración durante el programa MECESUP2 fue pobre en comparación con el total de proyectos adjudicados en sus tres convocatorias. Asimismo, dicha colaboración fue protagonizada por instituciones regionales del centro-sur del país y por grupos de unidades responsables de su ejecución cada vez más similares. Por otro lado, la tendencia a colaborar poco parece ser heredada de la primera fase del programa, lo que sugeriría una causa común. Frente a esta hipótesis es que se presenta a continuación una explicación tentativa tras el panorama colaborativo observado en proyectos MECESUP.

\section{Hacia una explicación tras el panorama colaborativo observado}

El análisis descrito en la sección anterior aborda tangencialmente el problema de la colaboración interuniversitaria como un fenómeno complejo. De hecho, lo hace al utilizar algunas herramientas de la Teoría de Redes acorde con el estudio de sistemas de este tipo.

A continuación, se presenta un análisis más profundo en esta línea, apoyado en la idea que la (pobre) colaboración observada podría ser un fenómeno emergente de un sistema (compuesto por universidades) de alta complejidad. Sin embargo, para desarrollar esta hipótesis es necesario definir una serie de conceptos previamente.

El primero de estos términos dice relación con la definición de "sistema complejo". En palabras simples, un sistema de este tipo es aquel compuesto por muchas unidades (universidades, por ejemplo) que interactúan de forma no trivial y poco intuitiva, dando lugar a propiedades emergentes ${ }^{7}$ universales del mismo tipo. Los sistemas complejos han sido estudiados desde hace ya varias décadas (Weaver, 1948), pero no es hasta la década de los ochenta, gracias al desarrollo de potentes computadores (Newman, 2011), que sus propiedades (emergentes) fueron descritas formalmente.

7 Propiedades que nacen de la interacción entre las unidades del sistema, pero cuyo diseño no está descrito en ellas. 
Con un enfoque apartado del reduccionismo, los sistemas complejos han sido estudiados con conceptos provenientes de la Física Estadística, los cuales se ocupan de las manifestaciones globales del sistema, dejando de lado aspectos particulares de las unidades que los componen. Desde esta mirada surgió con mucha fuerza hacia fines de la década de los noventa un método bastante intuitivo para estudiar esos sistemas complejos (Dorogovtsev, S. N. \& Mendes, J., 2002; Newman, 2003; Barabási, 2003; Barabási, 2009; Cárdenas, 2009; Solé, 2009). El método, utilizado también a lo largo de esta investigación, consiste en abstraer estos sistemas a un objeto matemático denominado grafo. Con ello, sus componentes pasan a ser nodos y sus relaciones, los enlaces entre estos. El grafo, entonces, constituye una representación estática, una "fotografía" de las relaciones entre las unidades que componen al sistema en un momento determinado (Urbina y Cárdenas, 2011; Cárdenas, Mouronte, Benito, Losada, 2010; Cárdenas, Mouronte, Santiago, Feliu, Benito, 2010; Cárdenas, Santiago, Tarquis, Losada, Borondo, Benito, 2010).

Ahora bien, uno de los aspectos más relevantes de las investigaciones acerca de los sistemas complejos tiene que ver con la evidencia de universalidad. Esto quiere decir que las propiedades que emergen en estos sistemas son ubicuas en términos estadísticos, independientemente de la naturaleza del sistema y, lo más llamativo, producto de la acción de reglas simples.

Si se pudiera definir en una palabra dicha ubicuidad estadística sería heterogeneidad. Los sistemas complejos (todos) son heterogéneos, desiguales. En términos matemáticos dicha propiedad se traduce en funciones de distribución, descritas por un escalado, llamadas Leyes de Potencia (Mitchel, 2009). En distribuciones de este tipo la dependencia de una variable respecto de otra obedece a una constante o a un factor de escalado denotando heterogeneidad, y donde no existe una medida típica (promedio) del sistema, es más, hay presencia de una amplia gama de valores, algunos muy alejados del promedio. Esto es totalmente distinto de lo que sucede en distribuciones que describen fenómenos aleatorios (triviales), en donde la gran mayoría de valores son cercanos al promedio y valores alejados de este son 
prácticamente imposibles de encontrar. Estos fenómenos aleatorios son descritos por la bien conocida distribución normal o gaussiana ${ }^{8}$ (Grinstead y Snell, 1997).

Lograr distribuciones heterogéneas requiere de organización, de cierto determinismo, o bien de reglas operando sobre el sistema. Por el contrario, la ausencia de reglas y la presencia de aleatoriedad son la clave para lograr distribuciones normales u homogéneas.

El origen de esta complejidad es atribuido a la evolución de los sistemas, además, es gratuita, no guiada ni buscada, según Stuart Kauffman (1993), sino producto de la autorganización de las unidades que conforman el sistema cuando son gobernadas por ciertos mecanismos o leyes fundamentales "ciegas" que la engendran. Esta es, precisamente, la hipótesis que manejamos respecto de la colaboración observada en este estudio.

Por ello, con el fin de profundizar en las causas de lo observado en esta investigación se diseñó una medida que permitiera caracterizar el sistema estudiado como un sistema complejo. De esta manera, y con la ayuda de datos e ideas aportados en entrevistas con especialistas, surgió la idea de aventurarse en la construcción de una "medida de complejidad" de las universidades participantes y su posible relación con la colaboración observada.

Desde hace bastante tiempo se ha intentado entender las organizaciones sociales desde la perspectiva de los sistemas complejos. Ya en 1961 Jacob expuso acerca de las razones tras el colapso de las grandes ciudades, haciendo una clara alusión a la responsabilidad de la complejidad respecto de esos procesos. Hoy en día, con un desarrollo sólido de las Ciencias de la Complejidad, se estudia desde esta perspectiva la vida de las empresas (Kauffman, 2000; Johnson, 2002) y las adaptaciones de instituciones públicas (Rouse, 2008).

Si bien intuitivamente se sabe que las instituciones son muchas veces sistemas que presentan complejidad, se trata solo de una

8 En honor a Carl Friedrich Gauss. 
apreciación cualitativa, ya que no existe una medida (cuantitativa) para definirlas. Esta fue la tarea que abordamos en el diseño de la medida de complejidad para las universidades participantes en proyectos adjudicados en colaboración dentro del programa MECESUP.

Seth Lloyd (2006) ha recopilado más de 40 medidas de complejidad: un grupo de ellas referidas a su organización; otras a la dificultad empleada para construirlas (tiempo, dinero, energía, etc.); y algunas medidas referidas a la dificultad para describir este tipo de sistemas.

Dentro de este último tipo destaca una medida de complejidad propuesta por el Nobel Murray Gell-Mann (1994) por ser una de las más intuitivas. Gell-Mann define la complejidad de un sistema como la longitud del mensaje más corto que lo describe empleando un lenguaje, método e interpretación que dos o más interlocutores comparten. Esta medida está estrechamente relacionada con la complejidad de Kolmogorov, (Gell-Mann, 1994; Kauffman, 2000) en donde el interés de esta última recae en la relación subjetiva entre la información y el observador, donde finalmente subyace la noción más intuitiva de complejidad. Fueron estos los conceptos utilizados para construir la medida de complejidad.

Como muchos sistemas, las universidades deben adaptarse a su entorno, procesando información del medio y adaptando su funcionalidad, razón por la que se les denomina sistemas adaptativos (Holland, 1975; Kauffman, 2000; Rouse, 2008; Mitchell, 2009). En los sistemas biológicos la diferenciación celular es un claro ejemplo de funcionalidades adaptativas. En sistemas sociales la presencia de comunidades (Newman, 2003; Castellano, Fortunato y Loreto, 2009) y el desarrollo de barrios comerciales y financieros en ciudades (Johnson, 2002) responden también a mecanismos de adaptación.

Para construir la medida de complejidad en esta investigación se tomaron como modelo aquellos sistemas complejos adaptativos más paradigmáticos: los seres vivos. Desde el punto de vista de la biología se puede decir que cuanto más complejo es un ser vivo, más información hay contenida en él. Así, quedan ligados los conceptos 
de complejidad e información, haciendo posible aplicar la medida de complejidad propuesta por Gell-Mann.

Ahora bien, para comparar la complejidad de los sistemas biológicos se necesitan ciertos criterios y el primero de ellos tiene que ver con el tamaño. Este criterio implica que a igual nivel de complejidad, un organismo más grande supone mayor información por un simple hecho cuantitativo. Evidentemente, el tamaño no basta para decidir si un organismo es más complejo que otro (puesto que la información podría hallarse "comprimida", a modo de estructuras más intrincadas) pero, por lo general, mayor tamaño implica mayor complejidad.

En segundo lugar existe un criterio estructural-funcional. Según este, para comparar el nivel de complejidad de una célula, por ejemplo, hay que observar sus organelos y su funcionalidad. Una célula eucariota contiene estructuras mucho más complejas que una célula procariota; es más, para reproducir una célula eucariota y su funcionalidad hace falta mayor información genética.

Por otro lado, todo ser vivo produce algo y dichos productos están estrechamente relacionados con su complejidad. Se puede considerar, entonces, un tercer criterio, aquel referido a la productividad del sistema. En este caso, mientras más y diversos productos elabore un sistema, más complejo será el mismo.

Por último, la evolución biológica gobernada por procesos selectivos y autorganizados (Kauffman, 1993, 1995, 2000) y plasmada en su historia filogenética también aportan complejidad a los seres vivos según un criterio evolutivo-selectivo. Así, un organismo se hará más complejo en su historia filogenética gracias a la simple acumulación de información en su genética durante el proceso evolutivo.

De esta forma, para el presente estudio estos cuatro criterios de complejidad biológica (tamaño, estructural-funcional, de la productividad del sistema y evolutivo-selectivo) fueron aplicados a las universidades para construir una medida de complejidad para ellas.

Tal como plantea Bernasconi (2006), la clasificación de las universidades ha sido un intento de organizar las instituciones de 
acuerdo con algunos atributos significativos que definan su perfil. El informe "Guiar el Mercado" (Brunner, 2005) y la clasificación propuesta por la Fundación Carnegie (Bernasconi, 2006) han permitido explorar diferentes puntos de vista desde los cuales se puede realizar una tarea como esta. No obstante, el enfoque aquí planteado es totalmente distinto, puesto que no propone una categorización de las universidades, tal como los trabajos antes mencionados, sino una medida de complejidad institucional para cada una de ellas.

La Tabla 4 presenta las variables consideradas para la construcción de la medida de complejidad propuesta para las universidades, en analogía con un sistema biológico.

Tabla 4: Criterios y variables para medir la complejidad de un sistema biológico y una universidad como sistema complejo adaptativo

\begin{tabular}{|l|l|l|}
\hline Criterio & Célula & Universidad \\
\hline C1 Tamaño & $\begin{array}{l}\text { Tamaño célula } \\
\text { Tamaño ADN }\end{array}$ & $\begin{array}{l}\text { Número de sedes } \\
\text { Número de alumnos }\end{array}$ \\
\hline C2 Estructural-Funcional & Pequeños órganos y sus funciones & $\begin{array}{l}\text { Ofertas de carreras pre-post grado } \\
\text { Cobertura de la oferta } \\
\text { Calidad académica }\end{array}$ \\
\hline C3 Evolutivo-Selectivo & $\begin{array}{l}\text { Filogenia } \\
\text { Ambiente selectivo }\end{array}$ & $\begin{array}{l}\text { Historia } \\
\text { Selectividad } \\
\text { Acreditación }\end{array}$ \\
\hline C4 Productivo & Productos (calor, desechos, etc.) & $\begin{array}{l}\text { Proyectos } \\
\text { Publicaciones } \\
\text { Tasa de titulación }\end{array}$ \\
\hline
\end{tabular}

Es necesario señalar que la medida de complejidad no busca hacer un ranking de calidad de las universidades, sino que su objetivo es caracterizar la distribución de ellas según la escala de complejidad, lo cual tiene implicancias totalmente distintas y que se detallarán más adelante. Asimismo, la medida propuesta fue aplicada solo a aquellas instituciones que participaron en colaboración en proyectos MECESUP, dejando de lado las universidades chilenas que no se adjudicaron proyectos colaborativos en este programa.

Ahora bien, considerando que la medida de complejidad requiere de un lenguaje y método consensuados, en este caso el lenguaje corresponderá solo al conjunto de variables descritas en la Tabla 4, en tanto que el método, a la siguiente ecuación,

$$
\text { Complejidad }=\alpha(C 1)+\beta(C 2)+\gamma(C 3)+\delta(C 4),
$$


donde los valores de los parámetros $\alpha, \beta, \gamma$ y $\delta$ corresponden a la ponderación de cada uno de los cuatro criterios tomados en cuenta. Según esta ecuación, todas las variables están relacionadas positivamente con la medida, lo que quiere decir que entre más alto es el valor de la variable, mayor es la complejidad que aporta al sistema.

Para definir la ponderación de cada uno de los criterios se construyó un grafo de similitud entre las universidades que colaboraron en proyectos MECESUP, utilizando las mismas variables de la Tabla 4 (como se expresa en la Figura 8). En dicha red aparecen ordenadas las universidades de menor a mayor, según los punteros del reloj y por tamaño y color de acuerdo con el grado de similitud con el resto. Así, los nodos más grandes y de color más cálido representan universidades que se asemejan más a las otras, en tanto que los nodos pequeños corresponden a universidades que son distintas de sus pares.

Figura 8: Grafo de similitud entre universidades colaboradoras en proyectos MECESUP2. El grado de similitud entre dos universidades está representado por el grosor de los enlaces. El tamaño de los nodos representa qué tan semejantes al resto de instituciones son las universidades estudiadas

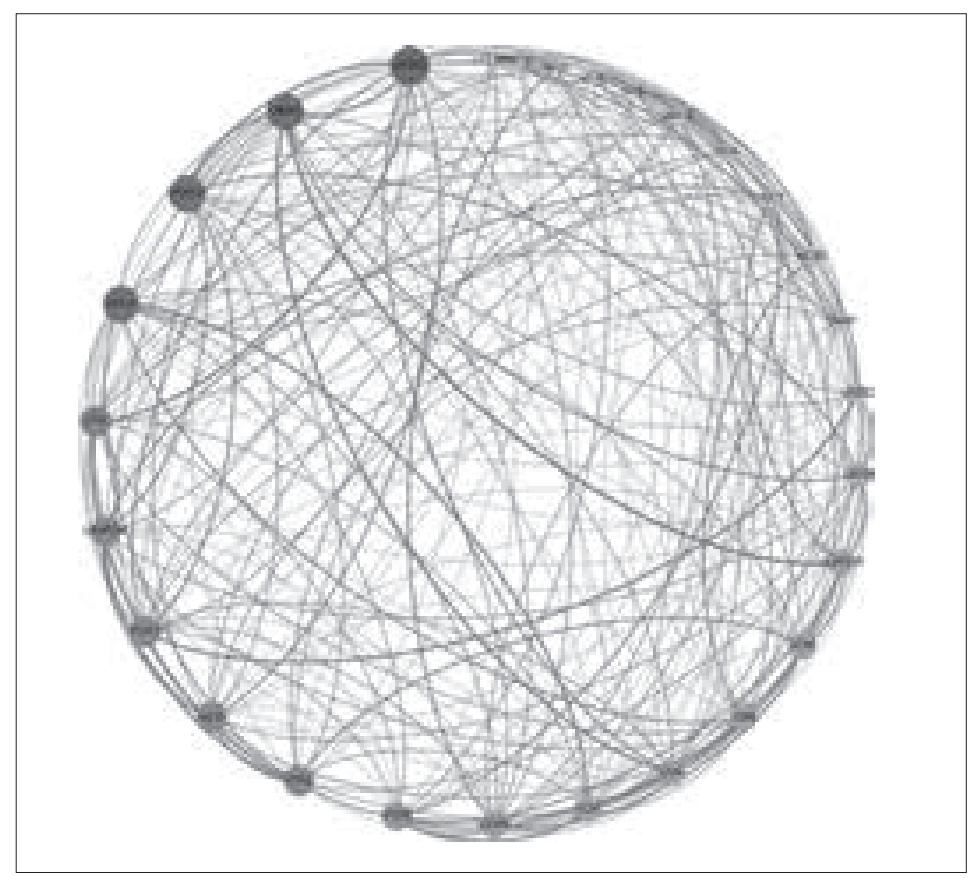


Como paso siguiente se estudió la distribución de frecuencia de las variables compartidas por las universidades. Con este análisis se detectaron variables, muy frecuentes en magnitud, entre un gran número de universidades y otras compartidas por pocas. Sobre la base de una de las definiciones de complejidad (Lloyd, 2006), aquella referida a la dificultad para la creación de un sistema, en esta investigación se asumió que aquellas variables semejantes en magnitud entre muchas universidades son "fáciles" de obtener y, por lo tanto, contribuyen menos a su complejidad. Por el contrario, aquellas que son semejantes entre un pequeño número de universidades son "difíciles" de obtener, contribuyendo más a la medida. La Tabla 5 muestra la ponderación de cada uno de los criterios según el análisis recién descrito.

Tabla 5: Valor de los parámetros utilizados para ponderar los criterios de complejidad

\begin{tabular}{|c|c|}
\hline Parámetro & Valor (\%) \\
\hline$\alpha$ & 10 \\
\hline$\beta$ & 37 \\
\hline$\gamma$ & 29 \\
\hline$\delta$ & 24 \\
\hline
\end{tabular}

Con estas ponderaciones para cada uno de los criterios se calculó la medida de complejidad de acuerdo con la ecuación 1 para cada una de las universidades bajo estudio. La escala de complejidad se muestra en la Figura 9.

Figura 9: Escala normalizada de complejidad para las universidades en estudio

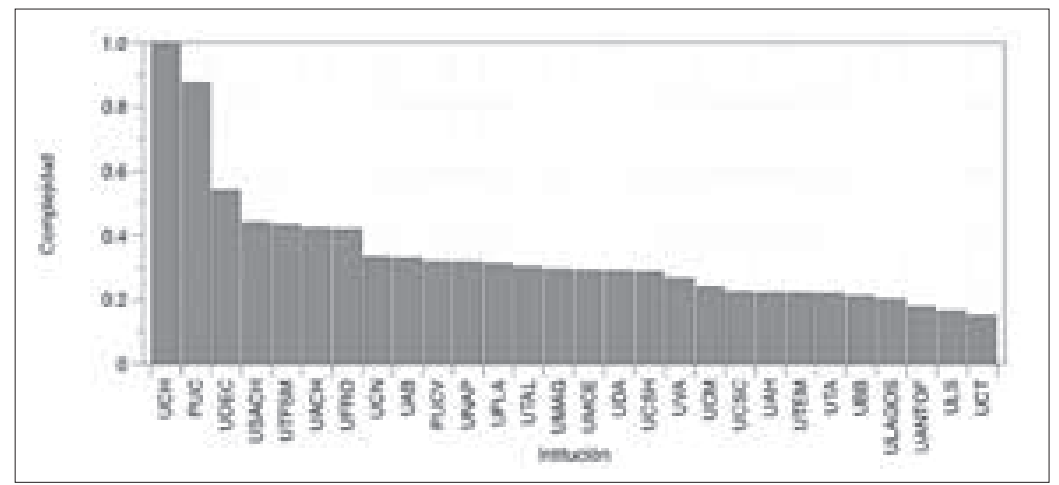


Según la medida propuesta, la gráfica pone en evidencia que dos de las universidades analizadas, UCH y PUC, tienen una complejidad muy superior a la del resto. Le sigue, más abajo, un grupo de universidades compuesto por la UDEC, la USACH y la UTFSM.

En un intento por organizar las universidades se analizó si es que a partir de la estructura de red presentada en la Figura 8 se podrían rescatar grupos (clusters) de universidades según su complejidad (la Figura 10 muestra que esto tiene sentido). Al ser estrictos a la hora de considerar dos instituciones como semejantes (i.e., eliminando aquellas variables en las que muchas universidades presentan un valor similar) se obtuvo un grafo con una estructura de comunidades muy representativo de la escala de complejidad de la Figura 9. De hecho, es posible observar que las universidades de mayor complejidad (la UCH, la PUC y la UDEC) pertenecen a una misma comunidad (nodos verdes). Lo interesante es notar que su semejanza con el resto es muy baja (nodos pequeños), lo que podría evidenciar que su alta complejidad viene dada por un carácter distinto del resto de las universidades. Otras comunidades, las de color púrpura, rojo y celeste, se corresponden con aquellas universidades de complejidad media-alta, media y baja, respectivamente.

Si bien las comunidades detectadas en el grafo de acuerdo con el algoritmo propuesto por Newman (2006) no tienen una correspondencia exacta con la escala de complejidad, es una clara evidencia de que el enfoque relacional de variables puede servir como un método para clasificar las instituciones, especialmente si se considera que utilizando este método la organización de los grupos de universidades emergió desde las características del mismo el sistema universitario estudiado. 
Figura 10: Grafo similar al de la Figura 8, pero con restricción en los enlaces y nodos agrupados por comunidad. El tamaño del nodo representa el grado de semejanza de este con el resto. El tono de los nodos representa la comunidad a la que pertenecen según el algoritmo de modularidad propuesto por Newman (2006)

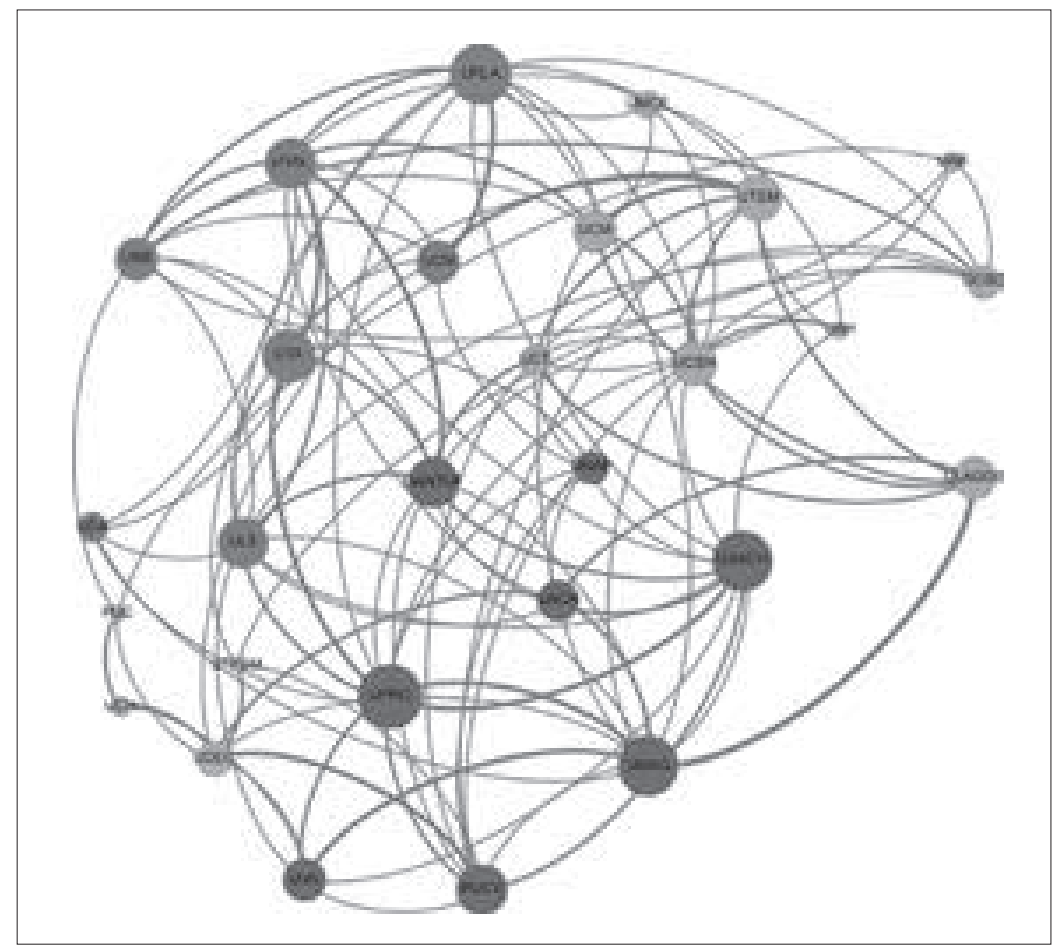

Por último, es preciso recalcar que los modelos propuestos para clasificar las universidades según complejidad (tanto el referido a la medida de complejidad como el de comunidades en el grafo de semejanza) requieren de mayor investigación y lo que aquí se presenta constituye solamente una primera aproximación al problema.

a) Sobre la complejidad de la universidades y la colaboración en proyectos MECESUP

Esta investigación detectó una conexión entre el nivel de complejidad institucional y la participación en proyectos colaborativos dentro del programa MECESUP. Las universidades que más colaboraron y las con mejor posición estratégica durante el programa en sus dos fases, correspondieron por lo general a aquellas universidades de 
complejidad alta y media de acuerdo con la medida propuesta. Solo en el año 2008, correspondiente al año de menor colaboración, las universidades que jugaron un papel protagónico en las redes de colaboración fueron aquellas de baja complejidad.

Asimismo, la medida de complejidad puede verse reflejada en la capacidad de las universidades para dirigir proyectos (Figura 11). Al observar el número de proyectos que dirige cada una se pudo ver que fueron aquellas de mayor complejidad las que con más frecuencia se responsabilizaron de la ejecución de proyectos en el programa MECESUP2.

Figura 11: Número de proyectos en colaboración dirigidos por universidad durante el programa MECESUP2. Las universidades están ordenadas de izquierda a derecha, según escala de complejidad

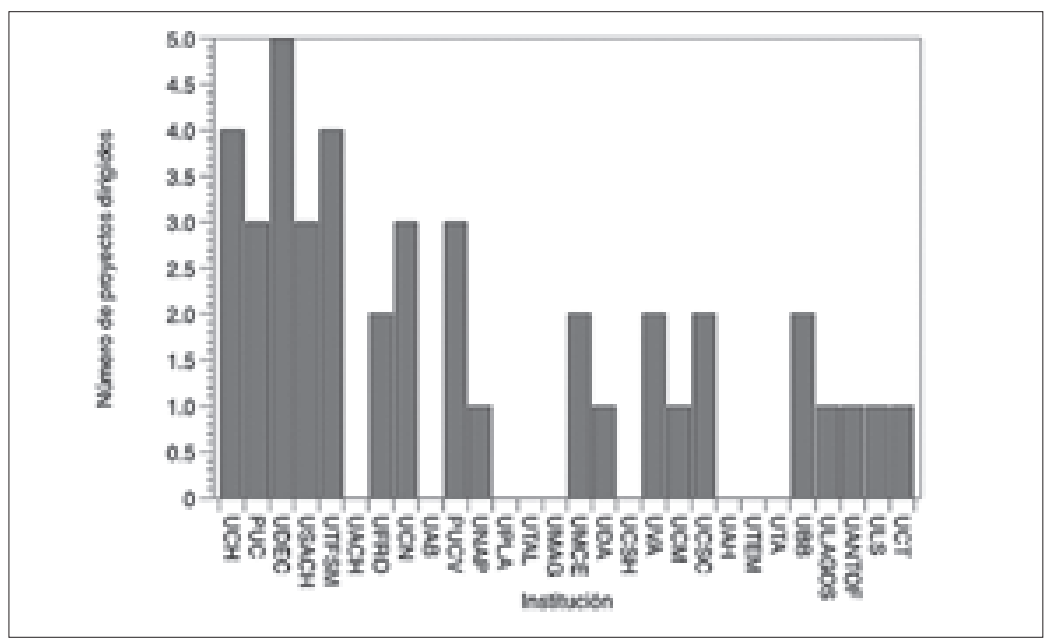

b) Una explicación tentativa para la (pobre) colaboración observada

De acuerdo con el informe elaborado por Suurla, Markkula y Mustarjarvi (2002) la colaboración entre universidades depende de la confianza recíproca para desarrollar una determinada tarea.

La confianza, en su más amplio significado, puede ser descrita como el grado de seguridad de que un dispositivo o sistema (por 
ejemplo una universidad) opere exitosamente en un ambiente específico durante un determinado período. Esta confianza se verá más o menos reforzada en función de las acciones, los actos previos y las posibles pruebas halladas y, por lo tanto, dependerá de la información disponible respecto de su desempeño, lo que para el caso aquí estudiado corresponde a la información que una universidad tenga sobre otras potenciales colaboradoras.

Desde esta perspectiva, lo que podría haber tras la colaboración entre universidades en proyectos MECESUP sería una decisión dependiente de dicha información. De esta manera, una institución que conoce sus capacidades y límites asumiría que un par "similar" podría desarrollar capacidades equivalentes o complementarias y tendría límites semejantes, con lo cual una institución "confiaría" en instituciones parecidas a ella: esa es la hipótesis que se desarrollará a continuación.

Lo anterior se apoya, además, en la evidencia que apunta a que los lazos sociales, de los cuales también emerge la complejidad, dependen de la homofilia (Kossinets y Watts, 2009; Cárdenas et al., 2010a), es decir, de ver en el otro a un semejante.

Si se observa el grafo de similitud entre las universidades estudiadas (Figura 8) lo que se aprecia es heterogeneidad, desigualdad. De hecho, instituciones como la UTFSM, la PUC y la UCH comparten pocas características con el resto. Por otro lado, la USACH, la UMAG y la UFRO comparten más cosas con el resto de las universidades. Notoriamente dos de ellas, la USACH y la UFRO, tienen papeles importantes de colaboración en las redes estudiadas durante MECESUP2, lo que avalaría la hipótesis aquí planteada.

La hipótesis se sustenta también en la teoría que demuestra que la heterogeneidad de los sistemas complejos puede nacer de una distribución heterogénea de las características de las unidades que los componen, como la que aquí se muestra para las universidades. Cárdenas et al. (2010a) demostraron cómo una población (cualquiera) con una distribución heterogénea de atributos tiene la potencialidad de formar una red también heterogénea cuando los enlaces dependen 
de la compatibilidad entre dichos atributos. En estas redes la gran mayoría está pobremente conectada, pero hay algunos pocos individuos de esa población con muchas conexiones.

Tal como se mostró en la sección anterior, la escala de complejidad para las universidades no es otra cosa que una proyección del grafo de similitud antes mencionado. Lo interesante es que cuando se observa la función de distribución de esta escala aparece el sello de los sistemas complejos: heterogeneidad, descrita por una función de distribución que sigue una ley de potencia (Figura 12).

Figura 12: Distribución de la complejidad de las universidades según ranking en la escala de complejidad propuesta. La línea representa el ajuste a una ley de potencia. Notar que la escala de ambos ejes es logarítmica

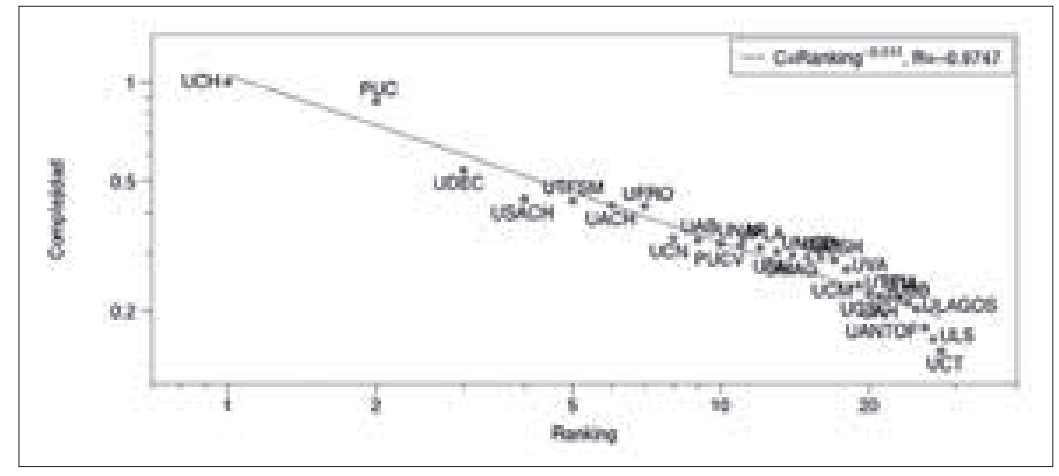

Este resultado, para nada trivial ni tampoco esperado, es signo inequívoco de la presencia de complejidad en el sistema estudiado, el que, de acuerdo con la hipótesis planteada, determinaría la colaboración: la heterogeneidad alimentaría la "desconfianza", dificultando la colaboración.

En un universo gaussiano en el que todas las universidades fueran similares, la Teoría de Redes (Erdös y Rényi, 1959) dice que sería muy probable que en todos los proyectos participara el mismo número de universidades, lo que claramente quedó descartado en esta investigación. Los resultados mostraron que lo más probable es encontrar universidades ejecutando proyectos individualmente, en tanto que lo menos probable es encontrarlas trabajando en dúos, más 
improbable aún en tríos y así sucesivamente. Lo destacable es que la función de distribución que describe la probabilidad de colaboración en proyectos MECESUP (Figura 13) corresponde nuevamente a una ley de potencia, denotando el carácter de sistema complejo que tienen las redes de colaboración. La similitud entre ambas fases tampoco es trivial y podría ser otra evidencia de que lo planteado en este trabajo es altamente probable.

Figura 13: Distribución de la probabilidad de colaboración, P(colaboración), de acuerdo con el número de universidades ejecutando un proyecto MECESUP. La línea representa el ajuste a una ley de potencia. Notar que la escala de ambos ejes es logarítmica

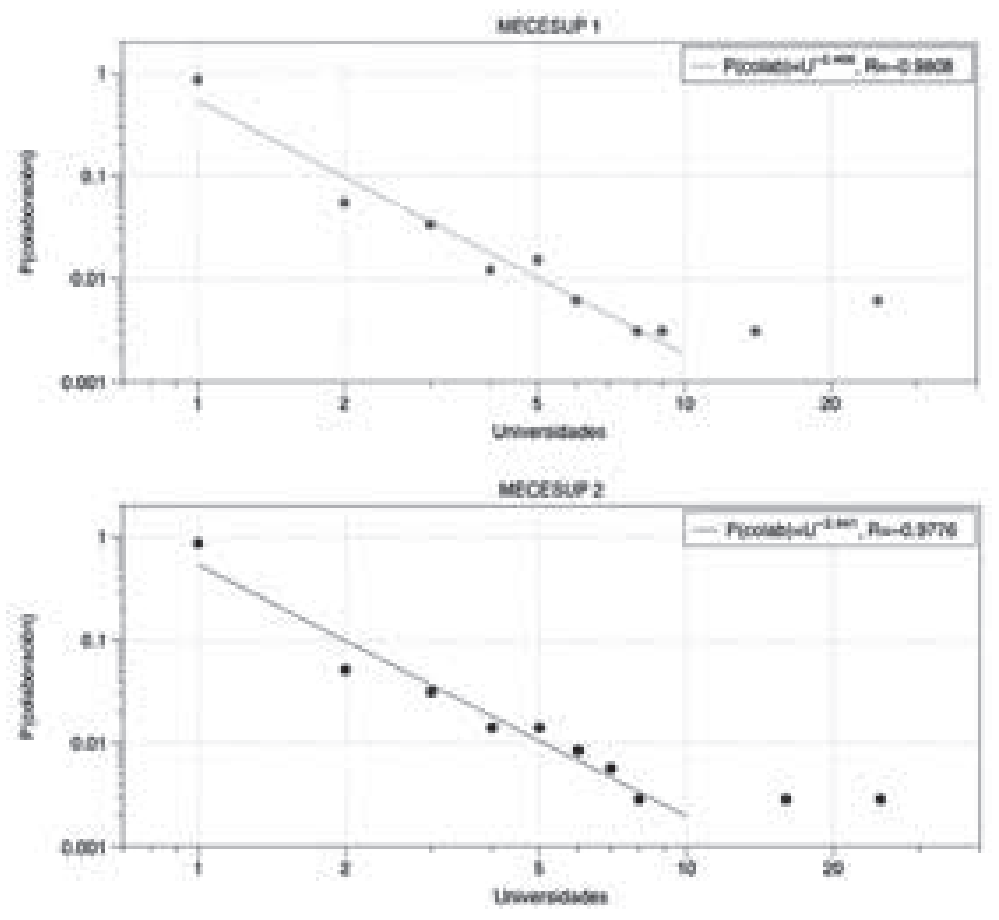

\section{Conclusiones}

En esta investigación se estudió la colaboración entre universidades a la luz de los proyectos MECESUP. Para esto se construyeron y analizaron redes de colaboración interuniversitaria y entre unidades responsables para cada año y eje temático del programa MECESUP2. 
Estos resultados fueron complementados con un análisis de las redes de la primera fase del programa. En forma paralela, se propuso una medida de complejidad institucional que permitiera dar una mirada desde las Ciencias de la Complejidad al problema de la colaboración entre universidades y generar hipótesis respecto del panorama descrito.

Las conclusiones de esta investigación pueden dividirse en dos partes. La primera de ellas en relación con el análisis de las redes de colaboración y su dinámica durante las dos etapas del programa MECESUP; la segunda, relacionada con las posibles causas tras la forma y niveles de colaboración detectados.

\section{a) Respecto del análisis de las redes de colaboración}

Las principales conclusiones de este análisis corresponden a dos hallazgos importantes: el primero de ellos se refiere al escaso número de relaciones de colaboración interuniversitaria en proyectos MECESUP durante las dos fases estudiadas; el segundo, a la disminución progresiva de esta (escasa) colaboración a través del tiempo.

Lo anterior no deja de ser un elemento relevante, pues es sabida la oportunidad para complementar capacidades que brinda el trabajo colaborativo. Con ello, lo deseable sería que las redes de colaboración, por lo menos, fueran transitorias en el tiempo (Sebastián, 2004), elemento que no ha sido observado, dada la disminución progresiva de la colaboración.

Los resultados mostraron que dentro de la pobreza colaborativa, ciertas universidades regionales fueron las que mantuvieron esa relación en los últimos años. Lo anterior resulta interesante, considerando que las redes regionales constituyen un importante instrumento para compensar los desequilibrios en la distribución de los recursos y las capacidades docentes (Sebastián, 2004).

Por otro lado, la colaboración entre universidades parece estar relacionada con la medida de complejidad institucional diseñada en este trabajo. De hecho, la UDEC y la UFRO, de complejidad media-alta según la medida propuesta, fueron muy activas en la colaboración 
al observar, de manera particular, los ejes temáticos durante 2006 y 2007. Algo parecido sucedió cuando se analizaron las redes totales de colaboración por año. En este caso, nuevamente las universidades de alta complejidad como la PUC y la USACH aparecían jugando un rol protagónico de colaboración. Esta correlación positiva entre complejidad institucional y colaboración se observó en ambas fases del programa MECESUP.

Los resultados mostraron, además, que esta medida de complejidad también se relacionaba con la capacidad de coordinar proyectos, puesto que fueron las universidades de mayor complejidad institucional las que con mayor frecuencia aparecieron como responsables de proyectos en colaboración.

A partir del análisis comparativo entre las dos fases del programa MECESUP, los resultados sugirieron que la segunda fase heredó los niveles de colaboración de la primera, sin embargo, fue distinta respecto del perfil de las universidades que protagonizaron dicha colaboración.

Del análisis de la colaboración entre unidades responsables de los proyectos, los resultados pusieron en evidencia que aquellas de carácter institucional fueron las de mayor presencia y que la diversidad de estas unidades ejecutando redes de colaboración fue cada vez menor durante el programa MECESUP2.

Por último, en este trabajo no se encontró una correlación entre la proximidad geográfica y colaboración como la que fue observada por Olmeda, Perianes, Ovalle, Guerrero, Anegón (2009) en su análisis de colaboración científica en España.

\section{b) Respecto de las causas tras la colaboración observada}

Los alcances de los resultados acerca de cómo la complejidad del sistema estudiado determinaría la colaboración pueden ser muchos, aunque el más importante de ellos se relaciona con las políticas para fomentar la colaboración futura entre instituciones en programas de este tipo. 
En efecto, si la hipótesis que se plantea es correcta (lo que parece bastante plausible considerando la evidencia mostrada en la Figura 12 y, en especial, en la Figura 13), cualquier medida que promueva la colaboración, pero que no considere la homogenización de las capacidades institucionales mínimas (algunos de aquellos criterios de mayor ponderación según las Tablas 4 y 5) se topará con este problema.

Muchas veces la complejidad no es algo deseado. Si bien puede ser útil para sistemas biológicos y tecnológicos, en sistemas que involucren directamente a personas, como el estudiado en esta investigación, puede tener implicaciones negativas, ya que la heterogeneidad se traduce generalmente en una propiedad indeseada: la desigualdad. La evidencia apunta a que la complejidad es espontánea en la naturaleza (Kauffman, 1993, 1995) y la heterogeneidad (su sello) aparece muy fácilmente cuando muchos componentes se relacionan gracias a ciertos mecanismos simples, como la homofilia, pero sin un control externo.

Es necesario decir en este punto que el problema aquí detectado asociado a la complejidad se refiere a la heterogeneidad que manifiesta el sistema de universidades estudiado y no a la complejidad institucional individual, lo que obviamente es algo deseado.

Por otra parte, la poca colaboración observada en proyectos MECESUP no se explicaría por los costos asociados a esta, sino que respondería, según la hipótesis planteada en este trabajo, a razones distintas relacionadas con el origen de los enlaces de colaboración. Algo parecido a lo detectado por García (2009) respecto del papel de la estructura formal de la universidad en la manera de participar en redes. De acuerdo con algunos académicos entrevistados y en concordancia con algunas investigaciones internacionales (Sebastián, 2004; Bercovitza y Feldmanb, 2011) si bien los costos de colaborar son reales, los beneficios asociados a la diversidad de conocimiento que se obtienen como resultado de ella representan un valor superior.

Los niveles de colaboración observados serían, pues, el reflejo de la desigualdad dentro del sistema universitario estudiado, la que 
no permitiría un mayor número de relaciones de colaboración para desarrollar un proyecto en red. Tal cual lo señala Sebastián (2004), una de las principales dificultades para la colaboración es la excesiva heterogeneidad de asociados, lo que se relaciona con asimetrías en sus capacidades y aportaciones, pudiendo dar lugar a la pérdida gradual del interés por parte de los participantes, con la consiguiente separación de algunos de ellos, o bien, la dilución de la red.

La homogenización del sistema universitario que fomentaría el trabajo en red no significa, sin embargo, una similitud de las instituciones a todo nivel, ya que esto resultaría prácticamente imposible e incluso indeseado, dada la oportunidad de complementar las capacidades que brinda la colaboración. Lo que esta investigación propone es considerar que, para fortalecer el establecimiento de redes de colaboración entre universidades, debieran nivelarse algunos aspectos básicos que promuevan la confianza en que el objetivo común será desarrollado de manera efectiva.

En esta investigación se han detectado algunos elementos clave como los más diferenciadores entre instituciones, entre ellos: la calidad académica, la cantidad y calidad de proyectos en ejecución y la productividad científica. De esta forma, las políticas para fomentar la colaboración entre instituciones debieran incentivar un crecimiento armónico de las universidades, que considere el sistema universitario al que pertenecen, que fortalezca la investigación en niveles cualitativos y cuantitativos parecidos y que asegure una calidad semejante de su cuerpo académico.

De esta manera, se propone la generación de políticas de fomento para la colaboración que apunten a mecanismos del tipo bottom-up, en los cuales las partes individuales, es decir, las universidades, se planifiquen con detalle, para luego enlazarlas formando componentes más grandes, los que a su vez se enlazan hasta formar un sistema educacional completo y de calidad. En este escenario menos heterogéneo, las relaciones de colaboración debieran emerger espontáneamente, sin la necesidad de premiarlas o impulsarlas desde arriba, fortaleciendo así las capacidades de las instituciones de educación superior para generar procesos de 
mejoramiento nutridos desde la cooperación interuniversitaria nacional e internacional.

\section{Referencias bibliográficas}

Barabási, A.-L. (2009). Scale-free networks: a decade and beyond. Science, 325 (5939), pp. 412-413.

Barabási, A.-L. (2003). Linked: how everything is connected to everything else and what it means. New York: Penguin Group.

Bernasconi, A. (2006). La difícil tarea de clasificar universidades. Calidad en la educación, (25), pp. 81-96.

Bercovitza, J. y Feldmanb, M. (2011). The mechanisms of collaboration in inventive teams: composition, social networks, and geography. Research Policy, 40(1), pp. 81-93.

Brunner, J. (2005). Guiar el mercado. Informe sobre la educación superior en Chile. Santiago: Universidad Adolfo Ibáñez.

Cárdenas, J.P. (2009). Modelo de redes complejas mediante enlace compatible. Aplicación a sistemas reales. Tesis Doctoral. Madrid: Universidad Politécnica de Madrid.

Cárdenas, J.P., Mouronte, M.L., Benito, R.M. y Losada, J.C. (2010a). Compatibility as Underlying Mechanism Behind the Evolution of Networks. Physica A, 389, pp. 1789-1798.

Cárdenas, J.P., Mouronte, M., Santiago, A., Feliu, V. y Benito, R.M. (2010b). Topological analysis of complex optical transport networks. International Journal Bifurcation and Chaos, 20, pp. 787-794.

Cárdenas, J.P., Santiago, A., Tarquis, A., Losada, J.C., Borondo, F. y Benito, R.M. (2010c). Soil porous system as heterogeneous complex network. Geoderma, 160, pp. 13-21.

Castellano, C., Fortunato, S. y Loreto, V. (2009). Statistical physics of social dynamics. Rev Mod Phys, 81, pp. 591-646.

Dorogovtsev, S. N. y Mendes, J. (2002). Evolution of networks. From biological nets to the internet and www. New York: Oxford University Press.

Erdös P. y Rényi, A. (1959). On random graphs. Publicationes Mathematicae, (6), pp. 290-297.

García, C. (2009). Análisis de redes de información y producción científica interdisciplinaria en organizaciones de educación superior: El caso de la Universidad Católica de Chile. Santiago: Consejo Superior de Educación. 
Gell-Mann, M. (1994). The quark and the jaguar: adventures in the simple and the complex. New York: W.H. Freeman.

Gershenson, C. (2012). The implications of interactions for science and philosophy. Foundations of Science, 10699-012, pp.1233-1821.

Grinstead, C. M. y Snell, J. L. (1997). Central limit theorem. En Grinstead, C. M., Snell, J. L. Introduction to probability (pp. 325-360). United States of America: AMS Bookstore.

Holland, J. H. (1975). Adaptation in natural and artificial systems. Cambridge, MA: MIT Press.

Jacob, J. (1961). The death and life of great American cities. New York: Random House.

Jarvis, P. (2006). Universidades corporativas. Nuevos modelos de aprendizaje en la sociedad global. Madrid: Narcea.

Johnson, S. (2002). Emergence. The connected lives of ants, cities and software. New York: Touchstone Press.

Kauffman, S. (1993). The origins of order. Self-organization and selection in evolution. New York: Oxford University Press.

Kauffman, S. (1995). At home in the universe. The search for the laws of selforganization and complexity. New York: Oxford University Press.

Kauffman, S. (2000). Investigaciones. Complejidad, autoorganización y nuevas leyes para una biología general. New York: Oxford University Press.

Kossinets, G. y Watts, D.J. (2009). Origins of homophily in an evolving social network. American Journal of Sociology, 115, pp. 405-450.

Lloyd, S. (2006). Measures of complexity a non-exhaustive list. Massachusetts: Massachusetts Institute of Technology.

MECESUP2 (2011). Datos de proyectos MECESUP desde 1999 a 2008. Disponible en www.mece2.com

Meyer, J., Ramírez, F. y Wotipka, C. (2008). Globalización, ciudadanía y educación: la eclosión de unos marcos de empoderamientos cosmopolitas, multiculturales e individuales. pp. 181-192. En Meyer, J.; Ramírez, F. (2010) La educación en la sociedad mundial. Teoría institucional y agenda de investigación de los sistemas educativos contemporáneos. Barcelona: Octaedro.

Mitchell, M. (2009). Complexity. A guided tour. New York: Oxford University Press.

Newman, M. (2003). The structure and functions of complex networks. SIAM Review, 45(2), pp. 167-256. 
60 LA COLABORACIÓN INTERUNIVERSITARIA EN CHILE. EL CASO DE PROYECTOS MECESUP DESDE LA PERSPECTIVA DE LAS CIENCIAS DE LA COMPLEJIDAD - C. Urbina, J. P. Cárdenas, D. Cárdenas

Newman, M. (2006). Modularity and community structure in networks. PNAS, 103, pp. 8573-8574.

Newman, M. (2011). Complex systems: a survey. Disponible en: http://arxiv. org/abs/1112.1440v1

Olmeda, C., Perianes, A., Ovalle, M, Guerrero, V. y Anegón, F. (2009). Visualization of scientific co-authorship in Spanish universities: from regionalization to internationalization. Aslib Proceedings, 61(1), pp. 83-100.

Rouse, W. (2008). Health care as a complex adaptive system: implications for design and management. Disponible en: http://medicine.utah. edu/internalmedicine/patient_empowerment/papers/Rouse $\% 20$ NAEBridge2008\%20HealthcareComplexity.pdf

Sebastián, J. (2004). Colaboración e internacionalización de las Universidades. Buenos Aires: Biblos.

Solé, R. (2009). Redes complejas. Del genoma a internet. Madrid: Tusquets.

Suurla, R., Markkula, M. y Mustarjarvi, O. (2002). Desarrollo e implementación de la gestión del conocimiento en el parlamento de Finlandia. C. Cantero, Traductor.) Helsinki: Oy Edita Ab.

Urbina, C. y Cárdenas, J. (2011). Análisis de redes sociales como una herramienta práctica para la mejora de la convivencia al interior del aula. Valladolid: VI Congreso Internacional de Psicología y Educación.

Weaver, W. (1948). Science and complexity. En American Scientist, 36(4). [Reimpreso en Klir, G. J. (1991) Facets of Systems Science]. 